\title{
The Problem of Uniqueness in Doppler Imaging
}

\author{
M. Al-Malki and Z. Eker \\ King Saud University, Science College, Department of Astronomy, P.O. \\ Box 2455, Riyadh 11451, Saudi Arabia
}

\begin{abstract}
The uniqueness problem in Doppler imaging is discussed. Most of the problem appears to be confusion between different meanings of the word "uniqueness". According to specific definitions presented in this study, there should not be any uniqueness problem.
\end{abstract}

\section{Introduction}

The Doppler imaging technique is able to produce spectacular maps showing the surface structures of cool spotted stars. This amazing fact stands out as a miracle, which forces the ultimate limits of the creative imagination of humanity since there is no telescope to resolve the surface structure of these stars. However this success of Doppler imaging lacks internal consistency in order to be proud and convincing, because of the uniqueness problem attributed to the Doppler images.

Today it can be accepted that the starspot hypothesis succeeds in reproducing observed line profile deformations within the observational uncertainties. Thus the first goal, which is to explain the observations, is fulfilled. But the current aim of spot models has developed a different course - primarily to generate information about the discrete surface feature that eventually will allow understanding of magnetic activities and dynamo mechanisms. Therefore current spot models are also expected to produce reliable spot parameters like sizes, shapes, locations, and temperatures of virtually existing spots. The reliability of such parameters is directly related to the problem of uniqueness.

If there are an infinity of solutions, that is, there are many different combinations of shapes, temperatures, locations, and sizes, which can provide the same effect on the line profiles, the maps produced by Doppler imaging would be worthless. Then proceeding to generate spot related information to understand stellar activity cycles, magnetic dynamo and their roles in stellar evolution would be impossible or speculative.

This study intends to show that the uniqueness problem associated with Doppler imaging is not fatal. Starspot hypothesis studied by Doppler imaging is no different from any other problem of science which depends on curve fitting.

\section{Discussion}

Vogt, Penrod \& Hatzes (1987) study image reconstruction by using the maximum entropy method, which involves finding an image with the greatest con- 
figuration entropy. A reconstructed image which is the simplest or smoothest is unique by definition. The maximum entropy method, therefore, allows one to obtain a unique solution. However, the uniqueness associated with this solution is often confused with the uniqueness of the true solution. Therefore, despite the fact that the maximum entropy method supplies a unique solution, Vogt et al. (1987) claim that there is, in principle, no unique solution because this simplest or smoothest solution is not guaranteed to be the true solution.

After describing why and how the maximum entropy method can supply a unique solution, Vogt et al. explain why a unique solution is not possible. Surely, casting the problem in matrix form, $I . R=D$, where $I$ and $D$ are the image and data vectors and $R$ is the transfer matrix between the two, the solution for the image vector is given by $I=D \cdot R^{-1}$. According to Vogt et al., the matrix $R$ has $\mathrm{n}$ columns, equal to the number of rows of $I$, and $\mathrm{n}$ is equal to the number of approximately equal area zones on the stellar disk. Also $R$ has $\mathrm{m}$ rows, equal to the number of rows of $D$, and $\mathrm{m}$ is equal to the number of observational data. Vogt et al. assumed $\mathrm{n}$ to be between 1200 and 2500 , whereas $\mathrm{m}$ is $500-1000$. Thus $R$ is not a square matrix, so the inverse of $R$ is not available. Also they believed that $n$ should be independent, where the number of observations is limited to $\mathrm{m}$. So there cannot be a unique transformation. Thus recovering the image vector I relies on iterations and trial-and-error between the image and the data space. A solution found by trial-and-error cannot be trusted to be unique, although this solution is guessed by choosing the simplest or the smoothest image (maximum entropy principles). According to them, there cannot be a unique transformation between the image and the data space. Even if there is one, the reconstructed image always inherits an uncertainty from data with a finite signal-to-noise ratio. One should not hope to recover the true image. Thus recovered images are not unique.

Mathematically speaking, one can not solve a problem if the number of equations $(m)$ is less than the number of variables $(n)$. If $m$ rows are independent, the number of area zones $(n)$ could be reduced to less than $m$. That will reduce the resolution of the reconstructed star image, which is acceptable due to the limited number of observational data. In other words, to obtain better resolution one needs more observations. Theoretically, to achieve maximum resolution, the zone number ( $n$ ) will be infinite, and the number of observations (m) should be also be infinite; in this case one can achieve the original image quality. Thus the observation number $\mathrm{m}$ can be also be independent.

However, the "vogtstar" test of Vogt et al. has been conducted by itself. The test image, which is the letters $\mathrm{V}-\mathrm{O}-\mathrm{G}-\mathrm{T}$, taken as being artificial spot images on the hypothetical "vogtstar", has been recovered uniquely and successfully from the pre-generated synthetic spectral lines, certainly well enough to recognize the letter-shaped spots and even some details to differentiate between the "O" and the "G" letters. Contrary to the non-uniqueness implied by their algorithm, Vogt et al. had to confess that the main features of the true image are well constrained by the data and do not rely heavily on entropy assumptions. They said, "unlike the traditional light-curve modeling approach which provides almost no unique information about spot shapes and location, Doppler imaging determines both rather accurately". 
Therefore, despite the annoying deformations caused by the uncertainty inherited from the data, the "vogtstar" test is convincing evidence that unique spot maps of spotted stars are attainable by Doppler imaging. To achieve the image quality of photographs may be a dream now, but it is not impossible theoretically.

\section{Conclusions}

The word "uniqueness", nevertheless, does not appear to have a unique meaning, which can not be confused or misinterpreted, among the discussions of published starspot models. There could be at least four different kinds of uniqueness:

1. Uniqueness of a final solution, from a best-curve-fit to the observational data.

2. Uniqueness of spot signatures on the line profile.

3. Uniqueness of the true solution (the recovered image being identical to the original image).

4. Uniqueness of photographic images, i.e. uniqueness which is not disturbed by the limitation of the image reconstruction problem.

According to above definitions, there should not be a uniqueness problem because:

1. It is always possible to achieve a unique solution for a curve-fitting problem, since this type of uniqueness is guaranteed by the methods, like leastsquares, maximum entropy, ILOT (Information Limit Optimization Technique) and c2 (or root-mean-square) minimization (Skilling \& Bryan 1984, Vogt et al. 1987, Budding \& Zeilik 1987, Lyon, Hollis, \& Dorband 1997). Therefore, according to first definition above, there should not be a problem.

2. There should not be a problem according to the second intended meaning of uniqueness, because the uniqueness of the spot signatures on the line profile has been confirmed by the "vogtstar" test (Vogt et al. 1987). Otherwise, it becomes impossible to explain why and how the original image is recovered at the end of iterations.

3. No one disagrees that there can not be a uniqueness problem according to the third intended meaning of uniqueness. By definition a recovered image is unique, if it is identical to the original.

4. On the other hand, the uniqueness of photographic images cannot be denied even though these recovered images lack small details within the limited resolution defined by the instrumentation and materials. Even fuzzy and disturbed images can supply unique information about the original object. Uniqueness can be a problem for photography only if no unique feature of the original image is recognized on a photograph. 
Obtaining a very true solution or an ideal image (identical to the original) always was, and always will be, impossible. But it is a mistake to identify this as a uniqueness problem.

\section{References}

Budding, E., \& Zeilik, M. 1987, ApJ, 319, 827

Lyon, R.G., Hollis, J.M., \& Dorband, J.E. 1997, ApJ, 478, 658

Skilling, J., \& Bryan, R.J. 1984, MNRAS, 211, 111

Vogt, S.S., Penrod, G.D., \& Hatzes, A.P. 1987, ApJ, 321, 496

\section{Discussion}

Cameron: I think you have expressed very nicely the essence of the "uniqueness" problem - in practice, you have to be very clear about the surface resolution you need in order to solve a given physical problem. The observer then has the responsibility to ensure that the data set has adequate phase sampling and signal-to-noise to give the required surface resolution. Given a good enough data set, the entropy constraint should be superfluous.

Al-Malki: It is a very good idea to work on in the future, but in general the more data we have from a star, the better the resolution we can achieve. Poor data means poor information. 